\title{
The distribution of insertionally polymorphic endogenous retroviruses in breast cancer patients and cancer-free controls
}

\author{
Julia H Wildschutte ${ }^{1,4}$, Daniel Ram², Ravi Subramanian', Victoria L Stevens ${ }^{3}$ and John M Coffin ${ }^{1 *}$
}

\begin{abstract}
Background: Integration of retroviral DNA into a germ cell can result in a provirus that is transmitted vertically to the host's offspring. In humans, such endogenous retroviruses (HERVs) comprise $>8 \%$ of the genome. The HERV-K (HML-2) proviruses consist of $\sim 90$ elements related to mouse mammary tumor virus, which causes breast cancer in mice. A subset of HERV-K(HML-2) proviruses has some or all genes intact, and even encodes functional proteins, though a replication competent copy has yet to be observed. More than 10\% of HML-2 proviruses are human-specific, having integrated subsequent to the Homo-Pan divergence, and, of these, 11 are currently known to be polymorphic in integration site with variable frequencies among individuals. Increased expression of the most recent HML-2 proviruses has been observed in tissues and cell lines from several types of cancer, including breast cancer, for which expression may provide a meaningful marker of the disease.

Results: In this study, we performed a case-control analysis to investigate the possible relationship between the genome-wide presence of individual polymorphic HML-2 proviruses with the occurrence of breast cancer. For this purpose, we screened 50 genomic DNA samples from individuals diagnosed with breast cancer or without history of the disease ( $n=25$ per group) utilizing a combination of locus-specific PCR screening, in silico analysis of HML-2 content within the reference human genome sequence, and high-resolution genomic hybridization in semi-dried agarose. By implementing this strategy, we were able to analyze the distribution of both annotated and previously undescribed polymorphic HML-2 proviruses within our sample set, and to assess their possible association with disease outcome.

Conclusions: In a case-control analysis of 50 humans with regard to breast cancer diagnosis, we found no significant difference in the prevalence of proviruses between groups, suggesting common polymorphic HML-2 proviruses are not associated with breast cancer. Our findings indicate a higher level of putatively novel HML-2 sites within the population, providing support for additional recent insertion events, implying ongoing, yet rare, activities. These findings do not rule out either the possibility of involvement of such proviruses in a subset of breast cancers, or their possible utility as tissue-specific markers of disease.
\end{abstract}

Keywords: Endogenous retrovirus, Provirus, HERV-K, Breast cancer, Betaretroviridae, MMTV, JSRV

\section{Background}

Breast cancer is the most common cancer and second most common fatal cancer among women in the United States. In 2014, according to American Cancer Society (ACS) estimates, 232,670 women will have been diagnosed with breast cancer and at least 40,000 women will have died

\footnotetext{
* Correspondence: john.coffin@tufts.edu

1 Department of Molecular Biology and Microbiology, Tufts University School of Medicine, 136 Harrison Avenue, Boston, MA 02111, USA

Full list of author information is available at the end of the article
}

from this malignancy in the United States [1]. It is the leading cause of cancer-related death in women of Caucasian, African-American, Asian, and Native American ethnicities, and is the most common cause of death in Hispanic women. However, the incidence of breast cancer varies with respect to ethnic populations, suggesting underlying genetic, environmental, or lifestyle influences in its development and/or progression [1,2].

In recent years there have been significant discoveries that have contributed to improved prevention and 
diagnosis of breast cancer. Most notable are the discoveries of the BRCA1 and BRCA2 genes, identified in multiplecase family studies in which breast cancer cases were observed to follow a Mendelian pattern of inheritance [3-5]. Subsequent family-based studies have failed to identify additional genes associated with increased breast cancer risk, although BRCA1 and BRCA2 account for just 20 to $40 \%$ of familial cancers and about $5 \%$ of all breast cancer cases worldwide [6]. More recently, large-scale genotyping and genome-wide association (GWA) studies have led to the identification of other breast cancer susceptibility loci [5,7-9], which are currently estimated to account for less than 2 to $10 \%$ of disease risk, leaving at least $50 \%$ of breast cancer risk that remains to be explained [10]. Although GWA studies have expanded key areas of breast cancer research, their nature is inherently self-limiting due to reliance on single nucleotide polymorphisms (SNPs). As a result, other sources and types genomic and structural variation -that are also polymorphic and inherited in Mendelian fashion- are excluded, including recently mobile genetic retroelements, leaving the possibility of their disease association closed to investigation in such analyses.

More than $8 \%$ of the human genome is recognizably of retroviral origin, representing the remnants of ancient germline infections from exogenous retroviruses [11]. During an active retroviral infection cycle, the viral genomic RNA is reverse transcribed into a double-stranded DNA copy that is then permanently integrated into the host genome. Thus, the integration of retroviral DNA into a germ line cell may lead to a provirus that is transmitted vertically to that host's offspring as an endogenous retrovirus (ERV) [12]. If such an integration event has no immediate negative affect to the host, the provirus may be passed successively from parent to offspring over generations, eventually gaining population-wide polymorphic persistence and even fixation within the population. The vast majority of human ERVs (HERVs) were formed from germline infection and integration tens of millions of years ago, having since become highly mutated and truncated, or recombined to form solo LTRs, and are thus present without any infectious or functional capacity. However, a small number of HERVs - particularly those having formed within the last few million years- have retained at least some coding capacity and many are actively transcribed in certain cancers as well as some normal tissues [13,14].

The most recent retroviruses to colonize the human germ line are from the betaretrovirus-like HERV-K (HML-2) group, most closely related to the exogenous mouse mammary tumor virus (MMTV) and Jaagsiekte sheep retrovirus (JSRV), which respectively cause breast cancer in mice and lung cancer in sheep [15-20]. Within the human genome, the HML-2 group of proviruses is represented by approximately 90 proviruses and about
1000 solitary LTRs [19]. Unique among HERVs, the HML2 group includes at least 23 human-specific proviruses, of which 11 are currently known to have polymorphic alleles of varying frequency within the population $[16,17,19,20]$. Genome-wide and population-based screens have provided a strong indication for the presence of other unique, polymorphic HML-2 proviruses within some humans, and additional insertions are likely to be identified in the nearfuture with improved genome sequencing technologies and population-wide detection strategies; however, research into the patterns and prevalence of such HERVs is lacking $[17,21]$. The possibility remains that members of this group are still capable of replication, either from very rare but still-active individual proviruses or from the formation of a replication-competent recombinant via complementation of expressed and co-packaged viral RNAs into a budding particle. In support of this possibility, most human-specific and all polymorphic HML-2 proviruses have more than one intact open reading frame (ORF), and some encode functional proteins and even retrovirus-like particles (RVLPs) [19,22-27]. Also, the rate of accumulation of HML-2 proviruses in the human genome appears to have been constant since the Homo-Pan divergence [21]. Although a naturally occurring HML-2 provirus with infectious capacity has yet to be observed, engineered consensus HML-2 proviruses are weakly infectious [28,29].

A growing number of reports continues to demonstrate increased levels of HML-2 transcripts and proteins in affected tissues from several types human disease, including but not limited to ovarian cancer [30], germ cell tumors [24,31-34], melanomas [35-40], and leukemias/ lymphomas [41,42]. Of particular interest has been HML-2 proviral expression in diseased tissues associated with breast cancer, with up-regulation of HML-2 both from breast tumor biopsies and cell lines derived from breast tumor tissues [41,43-48]. In matched-tissue analyses, spliced and unspliced HML-2 env transcripts have been detected in cancerous breast tissue, but not adjacent normal epithelia $[43,46,47]$. Also, the release of HML-2-encoded RVLPs associated with encapsidated, unspliced transcripts and RT activity has been shown for multiple breast cancer-derived cell lines [49-51]. While the consequence of endogenous HERV expression is poorly understood, an essential relationship may be inferred through the genetic association of an inherited provirus to a particular disease, as is known to occur in a few animal models, such as the association of certain MMTV proviruses and mammary carcinoma in mice $[52,53]$. Given their variable presence within the population and high levels of functional conservation, only the HML-2 group of HERVs contains representative candidates for such a scenario.

Two HML-2 proviruses, referred to as K113 and K115 (located respectively at chromosomal regions 19p12 and 
8p23.1) have been examined for possible disease association [54-57]. Present respectively within $\sim 30 \%$ and $\sim 15 \%$ of individuals tested, K113 and K115 are estimated to have integrated into the germline $<2$ mya and have functional ORFs $[19,20,57]$. At least one report has investigated the prevalence of K113 and K115 among breast cancer patients [54], however the prevalence of other polymorphic HML-2 proviruses has not been addressed. Furthermore, the presence of additional unique yet currently uncharacterized polymorphic HML-2 proviruses within the population [17] makes it difficult to conclusively test for a genetic association using conventional methods, such as microarray hybridization or genomic sequencing, which are essentially blind to the detection such unannotated genomic variation.

We report the distribution of polymorphic HML-2 proviruses, including elements not previously characterized, in a cohort of breast cancer patients and individuals with no history of the disease. In a combined approach using PCR screening and 'unblotting', or direct hybridization of DNA within semi-dried agarose, a high-resolution technique previously developed and used by our lab to characterize endogenous murine leukemia viruses $[14,58]$, we investigated the prevalence of individual polymorphic HML-2 proviruses in a case-control comparison. Although we found no significant difference in the prevalence of individual proviruses between groups, suggesting that common polymorphic HML-2 proviruses (present in $>5 \%$ individuals tested) are not associated with breast cancer. However, these findings do not exclude either the possibility that rarer HML-2 proviruses are somehow involved in a subset of breast cancers or will provide a meaningful biomarker of this disease.

\section{Results}

\section{Analysis of annotated polymorphic HML-2 proviruses in} breast cancer patients

We first sought to examine the prevalence of the currently described polymorphic HML-2 proviruses in a case-control analysis in order to determine whether any was detected with a strong difference in frequency between groups, and to provide a direct comparison for the subsequent analysis of previously uncharacterized polymorphic proviruses. For these purposes, we screened a panel of genomic DNA samples from diagnosed breast cancer patients and individuals with no history of the disease. Samples were generously provided by the American Cancer Society (ACS) and were from the Cancer Prevention Study II Nutrition Cohort (CPS-II). CPS-II is a large-scale study designed to provide a prospective means for investigating the relationship between lifestyle factors and exposure risk to cancer incidence, mortality, and survival [59]. We initially analyzed 50 unlinked and de-identified genomic DNA samples from breast cancer cases or controls ( $n=25$ per group).
Previous work from our lab and by others has led to the identification of 11 examples of HML-2 proviruses for which multiple alleles can be detected with varying frequencies among humans (Table 1) [15-17,19,20]. We verified the chromosomal locations for 8 of the 11 polymorphic proviruses within the February 2009 human genome build (GRCh37/Hg19), with reference to parallel BLAT searches against earlier genome builds (March 2006 Hg18; May 2004 Hg17; July 2003 Hg16). For a conventional and consistent nomenclature reference [19], the proviruses included here are identified by their chromosome location and position relative to other proviruses if multiple proviruses are present within the same chromosomal band. The full-length sequences of four elements are absent from all published builds: two proviruses, located at 10p12.1 (also referred to as K103) and at 12q13.2, are represented as solo LTRs; the 19p12b (K113) insertion site is empty, with no evidence of a polymorphic provirus at the site; the remaining provirus (referred to as K105) is integrated within the unassembled centromeric region Un_g1000219 and unaligned to the current genome build. However, the genomic regions flanking each integration site are publicly available (respectively JN675098.1, JN675106.1, JN675117.1, and JN675176) [19], and BLAT searches were performed to verify each chromosomal location.

Initial HML-2-specific PCR screening was performed with all CPS-II samples blinded and randomly sorted. Locus-specific amplification was performed to detect the alleles present at each HML-2 insertion site, with primers spanning either the 5' LTR of each provirus (indicating the presence of the more or less full-length allele) and spanning the integration site (to detect either a solo LTR or the ancestral pre-integration sequence) (Table 2). Representative products from each amplified site were sequenced in both directions to confirm the correct product and to ensure primer specificity (data not shown). Upon completion of the primary screen, information for the disease group (breast or prostate cancer) and case/control identity was unblinded, and the samples sorted and grouped accordingly. PCR amplification for each HML-2 integration site was repeated as above to confirm the initial results, and to provide a direct case-control comparison for the breast cancer sample group. The frequency of each provirus was calculated per site per group, and the results subjected to a $\chi^{2}$ analysis, with a $p$-value of $<0.05$ regarded as significant within the dataset. The results are summarized in Table 3.

The majority of HML-2 insertion sites examined had no significant difference in proviral frequencies between groups. However from our initial case-control screens, we observed the K115 provirus to be present at a higher prevalence within breast cancer cases $(6 / 25$, or to a frequency of 0.24$)$ than in the control group $(1 / 25$, or 
Table 1 Known polymorphic HML-2 proviruses in human DNA

\begin{tabular}{|c|c|c|c|c|c|}
\hline HERV-K notation & Locus & Start (bp) in $\mathrm{Hg} 19$ & Alleles $^{b}$ & Accession number & Reference \\
\hline & $1 \mathrm{p} 31.1$ & 75842771 & pro & AC093156.2 & {$[16]$} \\
\hline K106 & $3 q 13.2$ & 112743479 & pro, solo & AC024108.22 & [14] \\
\hline K109 & $6 q 14.2$ & 78427019 & pro, solo & AC164615.1 & {$[14,16]$} \\
\hline$K 108^{a}$ & $7 \mathrm{p} 22.1^{a}$ & 4630561 & pro, solo, tandem, pre & AC164614.1 & {$[16,26]$} \\
\hline K115 & $8 p 23.1$ & 8054700 & pro, pre & AY037929.1 & [19] \\
\hline \multirow[t]{4}{*}{ K103 } & 10p12.1 & 27182399 & pro, solo & AF164611.1 & [14] \\
\hline & $11 q 22.1$ & 101565794 & pro, solo, pre & AP000776.5 & {$[16,25]$} \\
\hline & $12 q 13.2$ & 55727215 & pro, solo, pre & JN675067 & {$[18,20]$} \\
\hline & $12 q 14.1$ & 58721242 & pro, solo & AC074261.3 & {$[16,25]$} \\
\hline K113 & $19 p 12$ & 21841536 & pro, pre & AY037928.1 & [19] \\
\hline
\end{tabular}

${ }^{a} \mathrm{~K} 108$ is present as a tandem provirus in the published genome with a single shared LTR in the middle. The start coordinate refers to the right provirus of the tandem pair.

${ }^{b}$ Pro, provirus; solo, solo LTR; pre, pre-integration (empty) site.

$0.04)$, with a $p$-value of 0.04 . On a preliminary basis, this observation was of interest, given the significant difference in frequency between groups for the sample size. However, this particular provirus has been previously analyzed for possible association with a few human diseases (including breast cancer [54]), without significant support. Thus, we attempted to test the observed difference within a larger collection of representative genomic samples (to $>90 \%$ statistical power). For this purpose, a unique set of 200 CPS-II samples (100 breast cancer cases and 100 controls) was analyzed for the presence of K115 alone. We found that the initial result was not corroborated in the repeat analysis, in which K115 was observed in 6/100 cases (0.06) and 11/100 controls (0.11) (corresponding to

Table 2 Primers and product sizes for the detection of polymorphic HML-2 proviruses

\begin{tabular}{|c|c|c|c|c|}
\hline Locus ${ }^{a}$ (synonym) & Forward $\left(5^{\prime} \rightarrow 3^{\prime}\right)$ & Reverse $\left(5^{\prime} \rightarrow 3^{\prime}\right)$ & Size $(b p)^{b}$ solo/pre & Predicted Bsr1 Fragment size (bp) \\
\hline $1 \mathrm{p} 31.1-1$ & AACTACGTGAAGAATGAAGA & AATAAAGCTGAGATAAGAGG & 1239 & 1752 \\
\hline $3 q 13.2-1$ & GCTCGGATTTCAACATCCAT & TCGTCCGACTTGTCCTCAATG & 1821 & 1985 \\
\hline $3 q 13.2-11$ & GCTCGGATTTCAACATCCAT & TATTGGTGACAGAGAGATGCAG & $1847 / 879$ & \\
\hline $6 q 14.1-1$ & TCGTCGACTTGTCCTCAATG & CTGCCAGTCTCAGGTGTTTG & 1075 & 1758 \\
\hline $6 q 14.1-\|$ & CCCCTGCTTATTGATGCTCTACG & TGAGGCTGAATGTGTGGAGTCC & $1526 / 556$ & \\
\hline 7p22.1a-1 & TACTGAACGATGCTGACGTTTGG & TTTGAACCATTATCACCCTA & 1407 & 2067 \\
\hline $7 p 22.1 b-T$ & GTCTGCAGGTGTACCCAACAG & TTTGCCCCATTATCACCCTA & 1216 & 1981 \\
\hline 7p22.1-II & CCTCCTGGTTCAAGGGATTCTC & GCTTTCGGGACTTCAACATTGG & $1387 / 419$ & \\
\hline 8p23.1a-1 & CTTGTGTTTCATTACAATCTATT & TTCAGTCATTCTATCATTAAGATTC & 1667 & 2513 \\
\hline 8p23.1a-II & CAGTCTATAGATGTGGATGCCT & AGCACTGAATCCAAACTCATAT & $1320 / 352$ & \\
\hline 10p12.1-I & CCACCATCTGAGAAGTGTGATG & AATGGAGTCTCCYATGTCTACT & 1342 & 1846 \\
\hline 10p12.1-II & CCACCATCTGAGAAGTGTGATG & GGCAACAAAGGGTTCATATGAGAA & $1508 / 540$ & \\
\hline $11 q 22.1-1$ & CCATGCTCAGAAAGGAAACA & TAGCTTCTTCCGAGCACACA & 1168 & 2076 \\
\hline $11 q 22.1-11$ & CCATGCTCAGAAAGGAAACA & ACCATCTGTCCTTCCACCAG & $1661 / 693$ & \\
\hline $12 q 13.2-1$ & CGGAGAATTCCACCTTCAAA & CTCGAGCGTACCTTCACCCTAG & 1377 & 2392 \\
\hline $12 q 13.2-11$ & CGGAGAATTCCACCTTCAAA & TGCATTGTGGTCATCCATTT & $1488 / 520$ & \\
\hline $12 q 14.1-1$ & GGAAACCCTTCCAACATTCCA & CCCCATTATCACCCTAGCTTC & 1299 & 1755 \\
\hline $12 q 14.1-11$ & GGAAACCCTTCCAACATTCCA & TGAGGCTGAATGTGTGGAGTCC & $1101 / 133$ & \\
\hline 19p12b-I & TGCATGGGGAGATTCAGAACC & TCGGGATCTCTCGTCGACTTGTCC & 1210 & 5287 \\
\hline 19p12b-II & TGCATGGGGAGATTCAGAACC & CGTGTTAGCCAGGATGGTCT & $310 / 1278$ & \\
\hline
\end{tabular}

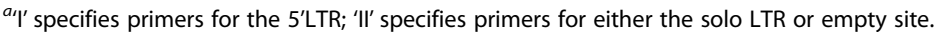

${ }^{b}$ Product sizes were estimated using in silico PCR (UCSC Genome Browser) of primer pairs. Product sizes for alleles for the 10p12.1, 12q13.2, and 19p12b proviruses were estimated manually by adding the distances to the distance to the nearest Bsrl site in the host genome regions flanking each integration site and in the respective provirus for that site. 
a $\chi^{2}$ of 1.61 and $p$-value of 0.20$)$. Collectively, these results suggest that no described individual polymorphic HML-2 provirus is associated with breast cancer occurrence for the CPS-II genomic samples screened; however these results do not exclude the possible association of HML-2 occurrence within a subset of breast cancer cases, or other disease types with implication for involvement.

Of the described polymorphic HML-2 proviruses, most are present in relatively high allele frequencies within humans ( $50 \%$ or above), and even the K113 and K115 proviruses are present in as many as $30 \%$ to $40 \%$ of tested individuals, depending on the ethnicity (on average, within $\sim 16-20 \%$ random individuals tested) [20,57]. Aside from the 11 described polymorphic integration sites, there is evidence that other unique polymorphic HML-2 proviruses are present in varying frequencies within humans $[14,16,17,20]$. However, the population distributions, genomic locations, and any sequence information of such elements are unknown. Previous work in our lab has shown that ERVs can be detected from fragmented genomic DNA by utilizing a high-specificity hybridization technique referred to as 'unblotting, during which restriction enzyme digested DNA is hybridized with a radiolabeled probe while immobilized in semi-dried agarose following electrophoresis $[14,17,58]$. Using this technique, polymorphic integrations can be identified as bands that vary between samples, and provides the means for direct comparison between individuals and/or groups. Therefore, we used unblotting to estimate the total number, distribution, frequency, and potential disease association of individual polymorphic HML-2 proviruses, including known integrations and those not previously described in the current genome databases, within our sample set.

\section{In silico analysis of polymorphic proviruses}

Initially, we performed in silico analysis as a means both to identify appropriate restriction enzymes for unblot analysis, and to generate predicted fragment patterns of previously annotated HML-2 proviruses with reference to the published genome sequence. For these purposes, we mined the Hg19 genome build for proviruses with high nucleotide identity to HERV-K113 (19p12b). We chose this full-length provirus as a reference since it is completely intact and represents one of the most evolutionarily recent germline integrations [19,20]. Full-length sequences were extracted for a total of 62 identified proviruses, to which 5 other described proviruses (located at $10 \mathrm{p} 12.1$ (K103), 19p12b (K113), at 12q13.2, and the K105 provirus located within an unaligned contig, Un_gl000219 [19] were manually added.

To identify a suitable probe sequence, we aligned and manually edited the full nucleotide sequences of all 66 proviruses, sorted individual elements in the resulting alignment by decreasing nucleotide identity to K113, and searched the alignment for sequence regions that were 1) highly similar among the most recently integrated elements (i.e., polymorphic and/or human-specific insertions), 2) distinct from the remaining proviruses, and 3) proximal to, but not within, the 5' LTR. We identified a highly conserved region of about $32 \mathrm{bp}$ within the gag leader region just outside of the 5 ' LTR and $\sim 1 \mathrm{~kb}$ from the start of the HML-2 consensus sequence (Figure 1). BLAT searches for this sequence returned 25 hits, all of which corresponded to HML-2 proviruses; 17 were identical to the queried 32 bp sequence, and 8 had two or fewer mismatches (Figure 1). Of note, the matching sequences included all described human-specific proviruses that

Table 3 Prevalence of polymorphic HML-2 proviruses in breast cancer

\begin{tabular}{|c|c|c|c|c|c|c|}
\hline \multirow{2}{*}{$\begin{array}{l}\text { HML-2 } \\
\text { Locus }\end{array}$} & \multicolumn{2}{|c|}{ Breast cancer cases $^{a}$} & \multicolumn{2}{|c|}{ Healthy controls ${ }^{a}$} & \multirow[b]{2}{*}{$\mathrm{x}^{2}$} & \multirow[b]{2}{*}{$p$-value ${ }^{b}$} \\
\hline & \# Positive & Frequency & \# Positive & Frequency & & \\
\hline $1 p 31.1$ & 16 & 0.64 & 17 & 0.68 & 0.09 & 0.76 \\
\hline $3 q 13.2$ & 25 & 1.00 & 25 & 1.00 & & \\
\hline $6 q 14.2$ & 21 & 0.84 & 23 & 0.92 & 0.75 & 0.34 \\
\hline $7 p 22.1 R$ & 25 & 1.00 & 25 & 1.00 & & \\
\hline $7 p 22.1 \mathrm{~L}$ & 24 & 0.96 & 25 & 1.00 & 1.02 & 0.31 \\
\hline $8 p 23.1 a$ & 6 & 0.24 & 1 & 0.04 & 4.15 & $0.04^{*}$ \\
\hline 10p12.1 & 24 & 0.96 & 25 & 1.00 & 1.02 & 0.31 \\
\hline $11 q 22.1$ & 23 & 0.92 & 20 & 0.80 & 1.49 & 0.22 \\
\hline 12913.2 & 20 & 0.80 & 21 & 0.84 & 0.13 & 0.72 \\
\hline $12 q 14.1$ & 23 & 0.92 & 22 & 0.88 & 0.22 & 0.67 \\
\hline $19 p 12 b$ & 3 & 0.12 & 3 & 0.12 & & \\
\hline
\end{tabular}

${ }^{a}$ Band sizes are based on estimated fragment lengths; each has been indicated by arrow in Figure 2 .

${ }^{b}$ Total sample size was 50 ( $n=25$ per group).

*Indicates significance ( $p>0.05$ ) within the dataset (not corrected for multiple comparisons). 


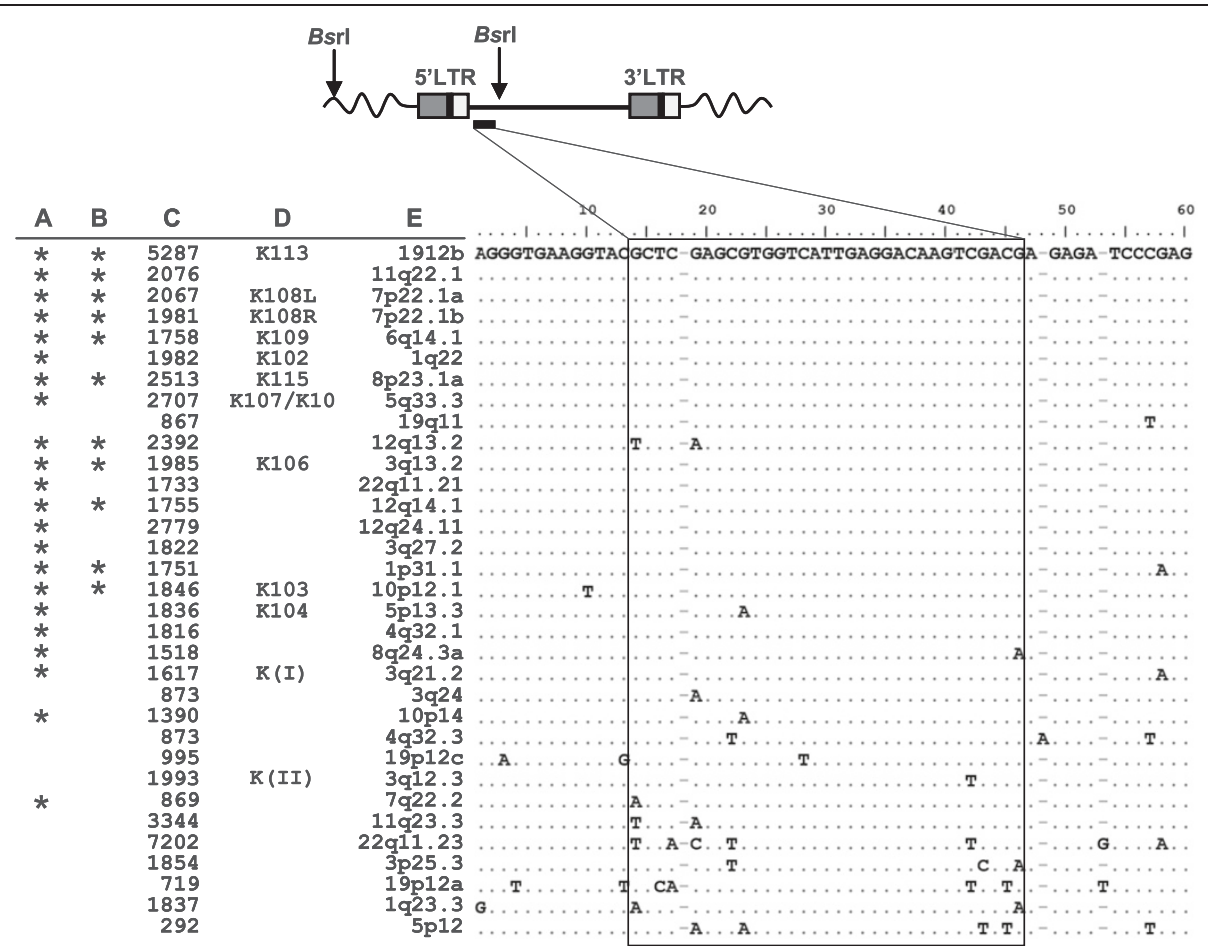

Figure 1 Identification of a conserved sequence for the detection of recently integrated HML-2 proviruses. A BLAT search of the 2009 human genome sequence build GRCh37/Hg19 for the $\sim 32$ bp K-seq sequence (shown in box) returned each provirus included in the alignment. The aligned sequences have been ordered with reference to percent identity to the K113 nucleotide sequence to depict the conservation of the region among the most recently formed germline integrations. Bases shared with $\mathrm{K} 113$ are indicated as dots, and differences are indicated by the base present at that site. Asterisks at left indicate $\mathbf{A}$. human-specific and $\mathbf{B}$. polymorphic proviruses; $\mathbf{C}$. predicted fragment sizes (in bp) based on restriction analysis of the published human genome ( $\mathrm{Hg} 19)$; D. reference aliases of each provirus; $\mathbf{E}$. chromosomal locus of each analyzed element.

are present within the Hg19 build, thus providing further support for the specificity of the probe. We therefore took advantage of this sequence, referred to here as 'Kseq', for in silico restriction fragment analyses and subsequent DNA hybridizations to facilitate detection of the most conserved HML-2 proviruses present within our sampled genomes.

An in silico restriction analysis for the Hg19 human reference genome build was performed to identify candidate restriction enzymes for hybridization of the Kseq site within sampled human genomes. Each of the 25 elements identified by BLAT of the Kseq region was simultaneously analyzed for enzymes predicted to cut at least once within the provirus but not within the 5'LTR, as well as for the nearest restriction site within the host flanking DNA. As a result, each Kseq-containing 'fragment' is predicted to contain a single proviral junction site, whereas the size of each fragment is defined by the distance from the first cleavage site 3' of the probe site to the nearest restriction site in host DNA (Figure 1, upper). Of about 35 candidate enzymes, 6 were analyzed in preliminary unblot screens using DNA from the T47D breast tumor-derived cell line (data not shown), and BsrI was finally selected for further hybridization screening with reference to overall fragment size distributions (ranging from $\sim 1 \mathrm{~kb}->6 \mathrm{~kb}$ ) and total number of fragments predicted to contain HML-2 proviral junction sites (as many as 30; discussed further below). The BsrI fragment distribution, as predicted from the Hg19 human reference build, is shown for reference in Figure 2A.

\section{Case-control analysis of polymorphic HML-2 proviruses in breast cancer}

Based on in silico predictions, we utilized unblotting $[14,58]$ to infer the distribution of polymorphic HML-2 proviruses within the genomes of the CPSII subjects. The unblotting technique is similar to Southern blotting DNA hybridization, but omits the transfer step of the DNA template, and consequently offers increased resolution without the loss of target DNA. A caveat is that at least $10 \mu \mathrm{g}$ of template is required per sample per run, thus challenging the examination under conditions of limited quantities of genomic DNA, as was for the CPS-II samples $(\sim 1 \mu \mathrm{g}$ per sample). We therefore subjected each sample to whole genome amplification (WGA; REPLI-g MIDI kit, Qiagen) step to generate working amounts of DNA per sample (at least $15 \mu \mathrm{g}$ in our hands). 


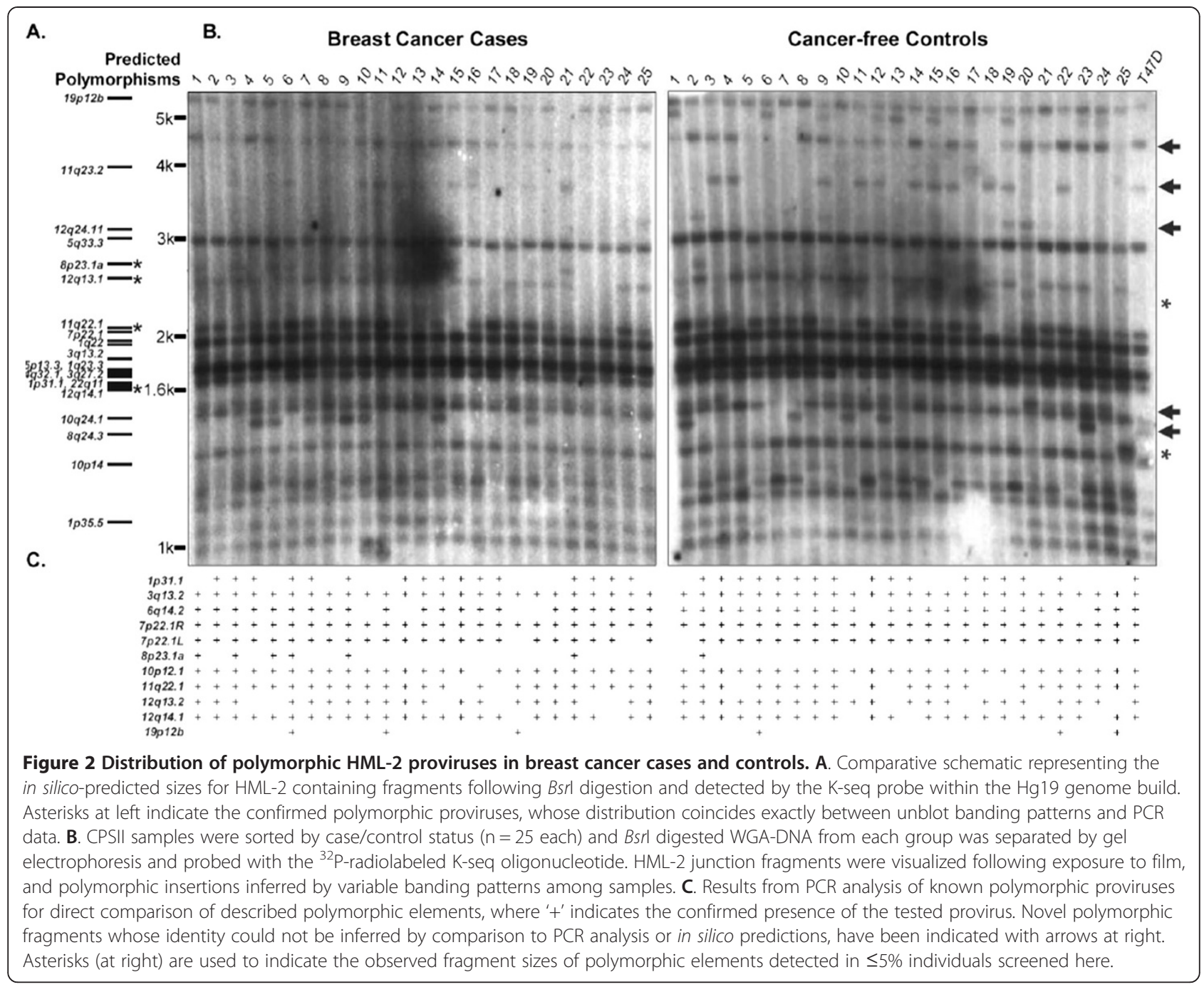

The amplified samples were individually digested with BsrI, and the products separated by electrophoresis through agarose. Simultaneous treatment of genomic DNA extracted from the T47D breast tumor-derived cell line was used as a control. The agarose was then dehydrated and the immobilized DNA hybridized with a ${ }^{32}$ P-labeled oligonucleotide complementary to the Kseq sequence, and finally exposed to film to visualize the prevalence and distribution of detectable HML-2-containing fragments. As with the initial HML-2-specific PCR screens of described polymorphic proviruses, all preliminary unblots were performed while samples were blinded, and following the subsequent release of their case/control status, the ublotting was repeated on a case-control basis and the samples analyzed by direct comparison between groups. Consistent with the cognate in silico analysis of the Hg19 human reference, unblots were interpreted such that each detected fragment represented a single proviral junction site, the size of which was dependent on the length of host sequence to the nearest 5' flanking restriction site. The resulting unblots are shown by case/control group in Figure 2B.

Overall, the banding patterns we observed by unblot of WGA-DNA were in close agreement with those predicted by in silico restriction analysis of the Hg19 genome build (Figure 2A and B), implying uniform amplification of all regions of the DNA. On average, we observed between 18 and 22 bands per lane in fragments that varied from sample to sample. To further characterize the fragments observed to be polymorphic among individuals, we compared the distribution of polymorphic HML-2 proviruses with that of previously described copies, as interpreted by PCR screen. Comparison between HML-2derived unblotting and PCR data allowed for the provisional assignment of a few fragments based on shared distribution between each analysis, each of which was further supported in agreement with the corresponding in silico predicted sizes (asterisks in Figure 2A). Most clearly represented were the fragments predicted to represent the 11q22.1 5'LTR junction, with a band around $2.1 \mathrm{~kb}$ that 
Table 4 Inferred case-control frequencies of previously undescribed polymorphic HML-2 proviruses in breast cancer

\begin{tabular}{|c|c|c|c|c|c|c|}
\hline \multirow{2}{*}{$\begin{array}{l}\text { Observed } \\
\text { band (bp) }\end{array}$} & \multicolumn{2}{|l|}{ Cases $^{b}$} & \multicolumn{2}{|l|}{ Controls $^{b}$} & \multirow[b]{2}{*}{$\overline{x^{2}}$} & \multirow[b]{2}{*}{$p$-value } \\
\hline & \# Positive & Frequency & \# Positive & Frequency & & \\
\hline 4600 & 25 & 1.00 & 22 & 0.88 & 3.19 & 0.07 \\
\hline 3700 & 10 & 0.40 & 11 & 0.44 & 0.08 & 0.78 \\
\hline 3200 & 1 & 0.04 & 4 & 0.16 & 2.00 & 0.16 \\
\hline 1500 & 25 & 1.00 & 23 & 0.92 & 2.08 & 0.15 \\
\hline 1470 & 8 & 0.32 & 5 & 0.20 & 0.93 & 0.33 \\
\hline
\end{tabular}

${ }^{a}$ Band sizes are based on estimated fragment lengths; each has been indicated by arrow in Figure 2 .

${ }^{b}$ Total sample size was 50 ( $n=25$ per group).

corresponded well with the expected distribution across all samples as determined by PCR (Figure 2B). Also near the $1.7 \mathrm{~kb}$ size, hybridized fragments were $100 \%$ consistent with the in silico size prediction and PCR distribution of the 12q14.1 provirus. Finally, we observed fragments matching the PCR distribution and size predictions of the $12 \mathrm{q} 13.2$ and K115 proviral junctions around $2.3 \mathrm{~kb}$ and $2.5 \mathrm{~kb}$, respectively. At least two of the hybridized fragments (located at 11q22.1 and 12q13.2) could be unambiguously assigned to the corresponding HML-2 provirus by locus-specific amplification and sequencing of their 5'LTRs and host flanking regions from template DNA obtained by elution from the corresponding unblotted gel regions (data not shown).

For the remaining known polymorphic HML-2 proviruses, discrimination of their specific locus was less clear by comparison with previous PCR analysis in conjunction with in silico predictions. A few such elements were fixed or nearly fixed within the sample set as indicated by locus-specific PCR, for example the proviruses located at $3 q 13.2$ and 7 p22.1b, thus complicating their assignment, however all CPSII samples were observed to have hybridized fragments near the predicted sizes of these elements (respectively 1985 bp and 1981 bp; also refer to Table 2). The expected banding patterns for the 1 p31.1 and $6 q 14.1$ elements could not be discerned by 5' LTR amplification, although the predicted junction fragments are around $1.7 \mathrm{~kb}$ (1751 for 1 p31.1 and 1758 for $6 \mathrm{q} 14.1)$. Given the number of bands that were both predicted and observed to fall within approximately the same size range, their specific banding patterns are likely to have been obscured. Another possibility is that some provirus-containing fragments may have been 'lost' due to the variable presence of common sequence polymorphisms within a meaningful $B s r 1$ site, or from sample-specific genomic structural variation in regions associated with HML-2 insertions; either scenario could potentially result in a junction fragment of an unexpected or undetectable size. Although this possibility cannot be excluded, we note the remaining predicted polymorphic HML-2 proviruses were consistent and wellsupported among all results from unblotting, PCR screening, and in silico restriction analysis.
To identify putatively novel integration sites, we examined each unblot for polymorphic bands that were neither predicted by in silico analysis of the described HML-2 polymorphic proviruses within the available databases, nor consistent with any distribution observed by direct PCR analysis. Several fragments, with estimated sizes from 1.4 to $4.6 \mathrm{~kb}$, were identified that met these criteria; these particular HML-2-containing fragments were clearly visible within multiple samples from either group, varying in frequency from $\sim 0.02$ to 0.98 . One such band of interest, specifically in lane 25 of the control group at $\sim 1.4 \mathrm{~kb}$, was represented by a single band not observed in any other sample, whereas the opposite was observed for other fragments, for example the band visible around $\sim 5.5 \mathrm{~kb}$, which was present in the majority of samples (each example is indicated by an asterisk near the relative fragment size in Figure $2 \mathrm{~B}$, right). In all, roughly $5-10$ polymorphic bands were visible, and of those, about 5 were clearly discernable across the total CPSII sample set (indicated in Figure 2B by arrows at right) The individual frequencies for each such fragment were directly compared between cases and controls by $X^{2}$ analysis (Table 4 ). Consistent with the initial PCR based analysis of described polymorphic elements as described above, no observed fragment differed significantly in its distribution between groups. The results indicate that at least within this sample set, polymorphic HML-2 proviruses do not imply association of a risk of breast cancer. However, our results also draw attention to an unexpected level of HML-2 content among these relatively few genomes tested in the present analysis.

\section{Discussion}

A few endogenous proviruses are known as causative to disease in experimental animal model systems, including the Betaretrovirus MMTV and mammary carcinoma in mice [15-20]. A similar association of the HML-2 proviruses, closely related to MMTV, is yet to be established, and remains a topic of study in the field. Here, we present our analysis of the distribution and prevalence of polymorphic HML-2 proviruses within the genomes of subsequently diagnosed breast cancer patients and from individuals with no history of the disease. For these 
purposes, we utilized two complementary approaches. We first developed a locus-specific PCR strategy to determine and assess the prevalence of each currently annotated polymorphic HML-2 locus with reference to the human reference database, as well as detection of the cognate unoccupied pre-integration sites and/or solo-LTR, where applicable. Secondly, we utilized unblotting, a high resolution and highly sequence-specific genome hybridization technique, as a means to provide direct inference of the prevalence and group distribution of putatively novel HML-2 polymorphic proviruses among the sampled genomes. For such proviruses, virtually nothing is known in terms of integration site, proviral structure, or functional features. To out knowledge, this is the first and most thorough report of such a comparison, and by far the largest representative set of human genomes analyzed for uncharacterized polymorphic proviruses from the most recently active HERV group.

The K113 and K115 proviruses were the first polymorphic HML-2 members to be discovered for which the empty-pre-integration site was still present within the population, and for which the proviral alleles were at relatively low frequencies, implying relatively recent germline integration (roughly estimated at $<200,000$ years and $\sim 1.2$ mya, respectively) [20]. In multiple reports, specific attention has been given to these two proviruses as possible candidates for roles in human diseases, including breast cancer [54], multiple sclerosis [56,57], schizophrenia [60], and autoimmune diseases [55,57]. Two of these reports are worth noting, in the context of the results presented here. In 2005, Moyes et al. [57] reported a "significantly" higher prevalence of the K113 provirus in the genomes of 109 multiple sclerosis patients. However, the analysis included multiple comparisons in terms of both proviruses tested and number of disease states, and the association was not replicated in a larger scaled study specifically addressing K113 prevalence and multiple sclerosis [56], highlighting the importance of being able to test such an initial finding on a statistically supported scale. Also pertinent is the 2004 report from Burmeister et al., in which K113 was observed at a somewhat higher frequency in individual breast cancer patients from an initial screen of 102 patients' genomes [54]. This particular result lacked statistical significance and was not further tested in larger screens. In the present study, our initial observation of a higher prevalence of the K115 provirus to breast cancer cases was not replicated in an independent set of samples, which we were fortunate to have been made available to us through the ACS CPSII Nutrition Cohort Study. Given the negative outcome of the PCR analysis of the second, larger sample set, the necessity for such added analysis is made clear.

In previous investigations for evidence of disease association, frequencies of the K113 and K115 proviruses have been reported to range from $\sim 10-20 \%$ for $\mathrm{K} 113$ and $\sim 5-12 \%$ for $\mathrm{K} 115$ [20]. Our results are consistent with these observations, with the exception of the K115 provirus in $\sim 24 \%$ of cases in the initial screen (Figure $2 B$ and $\mathrm{C}$ ). This frequency is not completely unexpected, however, as values as high as $>40 \%$ have been reported, depending on the race of the samples tested [20,57]. Similarly, in other analyses the K113 provirus has been observed at levels as high as $\sim 30 \%$, again depending on race $[20,57]$. Given such variance, the observed frequencies of the K115 provirus among DNAs from breast cancer cases may reflect an uneven representation with regards to ethnicity in the sample set. Alternatively, the higher frequency of K115 we observed in cases could be due to stochastic effects from the relatively small sample size used for the present analysis. As the samples were de-identified, we can only speculate on the factors, if any, influencing the observed distribution.

To date, all reports that have attempted to detect a genetic association of individual HML-2 proviruses have had a predominant focus to $\mathrm{K} 113$ and $\mathrm{K} 115$, given their status as the most recently integrated and conserved HML-2 loci known, however their analysis (over several diverse populations and disease groups) have offered little support for any implications in disease. This is perhaps not surprising, as a provirus that did have negative effects to the host would have a much reduced probability of population fixation, or would likely be removed from the population by selection. Thus, those proviruses with rare frequencies among humans would be more appropriate candidates for inference of disease-associated loci. The detection of such elements will necessitate much larger sample sizes than have been used to date, including the analysis presented here. Repeated searches for a disease association with one or two particular elements alone, such as has been the case for the K113 and K115 proviruses, will likely have similar outcomes as have been observed. We attempted to overcome such limitations by screening human genomes from the CPS-II ACS Nutritional Cohort using a highly specific DNA hybridization in a case-control comparison; we interpret our data to indicate the detectable presence of several as-yet-uncharacterized polymorphic proviruses, though none infer genetic association to disease.

We note that, although "new" bands observed from the unblots have a high likelihood of representing HML-2 containing genomic fragments, they may not reflect previously undescribed proviruses. For example, they could possibly have been a consequence of single base changes in known proviruses that destroyed or created a target sequence for BsrI restriction enzyme cleavage, Furthermore, the absence of certain bands in some samples could result from known full-length proviruses that have recombined to form solo LTRs in some individuals, or from recombination-mediated structural variation with reference 
to the human Hg19 build that would be undetectable in our approach. Also, a point mutation could lead to the generation of a new restriction site, for example within the 5 ' LTR, that would prevent the detection of the corresponding junction fragment by the probe. We searched for such an example from the fragments that we could tentatively identify as described HML-2 (asterisks in Figure 2A), and found the PCR and unblot data were in agreement, giving support that the BsrI target sites for these particular elements have not been disrupted. However, without knowledge of the chromosomal site of integration for each detected fragment, it is difficult to exclude the possibility of mutation (or possibly common SNPs among subjects) having occurred at restriction sites proximal to other detected proviruses.

In this study, we have developed an approach to identifying novel polymorphic proviruses in human populations, starting with samples of nanogram quantities of DNA, and we have provided evidence for a number of polymorphic proviruses that vary in frequency among the samples tested, some of which are present at quite low frequencies (for example, in lane 25 of the 'undiagnosed controls' in Figure 2B, asterisk at right). For the $\sim 50$ genomic DNAs in this analysis, between 18 and 22 bands were observed per sample. In the total set, there were about 10-15 junction fragments for which a corresponding known provirus could not be inferred from comparison to in silico or PCR analyses. Given the sample size, it is likely that at least some of these HML-2-containing fragments represent recent bona fide proviral integrations, which are present in just a portion of individuals, as would be predicted for such sites. At least two fragments, of sizes around $2.2 \mathrm{~kb}$ (in undiagnosed controls, sample 20) and $1.3 \mathrm{~kb}$ (same group, sample 25) (also asterisked in Figure 2B, right) appear to be present in less than $~ 5 \%$ of the total number of samples - $\mathrm{a}$ far lower representation than seen for any other described polymorphic provirus or previous report $[16,17]$. If not represented by solo LTRs in other individuals, such a provirus is likely to have been recently integrated and to closely resemble the original infecting virus, and, we can speculate, might also exhibit retained competency for replication. Current and future efforts to identify and characterize such novel proviruses will likely help in clarification of disease and/or phenotypic association of such sites.

\section{Conclusions}

In this study, we investigated the possible relationship between the genome-wide presence of polymorphic HML-2 proviruses in 50 humans with regard to breast cancer diagnosis. Although preliminary PCR analysis indicated the possibility of an elevated prevalence of one particular provirus, K115 (located at 8p23.1), in cases compared to controls and supported in in DNA hybridization screening, the observation was not replicated to a statistically significant level. Thus, we find no difference in the prevalence of proviruses between groups, suggesting that common polymorphic HML-2 proviruses are not associated with breast cancer in the tested individuals. These findings do not exclude either the possibility that rare HML-2 proviruses are involved in a subset of breast cancers, or their possible utility as tissue-specific expression and/or HML-2-derived products as potential marker(s) of disease. Interestingly, our findings do indicate a relatively high level of putatively novel HML-2 sites within the population, providing support for additional relatively recent insertion events and implication for ongoing activities. With continued improvements in sequencing technologies and in the detection of such elements, it is likely novel HML-2 polymorphic loci will be identified in the near-future; their genome-wide implications in terms of populationlevel structural variation and/or outcome phenotypic effects will remain, until then, to be seen.

\section{Methods}

\section{Human DNA samples}

Human genomic DNA samples were from the ACS Cancer Prevention Study II Nutrition Cohort (CPS-II), a prospective study of cancer incidence of approximately 184,000 Americans [59]. Nutrition Cohort participants, who were from 21 states and ranged from 50 to 74 years old at enrollment in 1992 or 1993, completed a mailed questionnaire that included questions on demographics, diet, and other lifestyle factors. Incident cases reported via questionnaire response were verified through medical records, linkage with state cancer registries, or death certificates. Blood samples were collected from a subset of Nutrition Cohort participants (21,965 women and 17,411 men) between June 1998 and June 2001, fractionated and stored in liquid nitrogen vapor phase at $-130^{\circ} \mathrm{C}$ until needed for analysis. All aspects of the CPS-II Nutrition Cohort study were approved by the Emory University Institutional Review Board (Atlanta, GA). Original CPS-II samples provided by the ACS were 100 total: 50 samples were from participants who were later diagnosed with breast cancer, and controls ( $\mathrm{n}=25$ per group); 50 samples were from participants who were later diagnosed with prostate cancer, and controls (also 25 per group). Controls were from participants who were cancer free at the time of diagnosis of the matching case. Samples were blinded, and subsequently unblinded following initial PCR analyses. All samples were deidentified, with information limited to case/ control assignment. To account for multiple comparisons, secondary PCR screens were performed with an additional 200 genomic DNA samples from the CPS-II cohort ( $\mathrm{n}=100$ per breast cancer cases or controls). As above, all samples were deidentified, and case/control information unblinded following PCR screening. 


\section{Whole genome amplification}

To obtain sufficient DNA for unblotting and PCR analyses, individually screened CPS-II DNA samples $(\sim 1 \mu \mathrm{g})$ were subjected to whole genome amplification (WGA). WGA was carried out according the manufacturer's protocol (MIDI Repli-G, Qiagen) with a starting volume of $5 \mu \mathrm{L}$. Briefly, $\sim 40 \mathrm{ng}$ genomic DNA per sample was denatured and neutralized using the supplied buffers in volumes of $5 \mu \mathrm{L}$ and $10 \mu \mathrm{L}$, respectively, for 3 min each at room temperature (RT). A mixture containing buffered \$29 polymerase (MIDI Repli-G, Qiagen) and random hexamers was added to each sample for a final volume of $50 \mu \mathrm{L}$ and the samples incubated $16 \mathrm{hr}$. at $30^{\circ} \mathrm{C}$. Amplified DNA was extracted using $2 \mathrm{~mL}$ heavy phase-lock gel tubes (5 PRIME) in $200 \mu \mathrm{L}$ volumes according to the manufacturer's protocol. DNA was precipitated from the aqueous phase in $95 \%$ ethanol $+100 \mathrm{mM} \mathrm{NaOAc}$, pH 5.2 to a final volume of $1 \mathrm{~mL}$ and incubated overnight at $-20^{\circ} \mathrm{C}$. The WGA DNA was pelleted at $14,000 \mathrm{rpm}$ for $30 \mathrm{~min}$. at $4^{\circ} \mathrm{C}$, washed in $1 \mathrm{ml}$ cold $70 \%$ ethanol, the centrifugation repeated, and the ethanol carefully aspirated. Pellets were dried $30 \mathrm{~min}$. at $37^{\circ} \mathrm{C}$, resuspended in $100 \mu \mathrm{L}$ sterile water, and the WGA DNA measured using a NanoDrop spectrophotometer.

\section{PCR amplification}

For 11 loci with evidence of multiple alleles including the provirus form, locus-specific primers were designed to amplify the 5' LTR of the provirus at each site using the most recently updated human genome Hg19 reference build (Table 1). For each locus, a primer was designed within $\sim 2 \mathrm{~kb}$ of the provirus edge within the flanking DNA of the host, and a second primer within the proviral leader sequence, outside of, but near, the 5 ' LTR. A third primer was designed in the host DNA downstream of the integration site in order to detect and differentiate the remaining alleles, including solo LTRs and unoccupied integration sites. Primers were designed using Primer3 v.0.4.0 and obtained from IDT, unless otherwise noted. An in silico PCR (UCSC Genome Browser) was used to estimate target amplification and product size for each primer pair, as provided in Table 1. All PCRs were carried out using 200 ng WGA DNA as template with 1.5-2.5 $\mu \mathrm{M} \mathrm{Mg}^{++}$, $200 \mu \mathrm{M}$ dNTPs, $0.2 \mu \mathrm{M}$ each primer, and 2.5 U Platinum Taq Polymerase (Invitrogen). 10 uL of each PCR reaction were analyzed by electrophoresis through $1 \%$ agarose in $1 \times$ TBE. Products from 2 separate positive PCR reactions per primer set were sequenced to confirm the desired product.

\section{In silico restriction analysis}

We used an in silico approach to identify useful restriction enzymes for subsequent DNA hybridizations to visualize HML-2 proviruses, and to generate a restriction fragment comparison from existing genome sequence data for reference during unblotting (see below). The HERV-K113 sequence (AY037928) was analyzed for restriction enzymes predicted to cut at least once within the provirus but not within the 5'LTR (NEBCutter2.0), for a total of 36 candidate enzymes. Simultaneously, we mined the 2009 human genome build (GRCh37/hg19) for proviruses with high percent identity to HML-2, again using the K113 nucleotide sequence as a reference. For the 32 proviruses identified from the search, we performed an in silico restriction analysis as follows. About $5 \mathrm{~kb}$ of sequence was extracted in both directions from the start of the 5'LTR. Each sequence was 'digested' in NEBCutterV2.0 for each of the 36 restriction sites with reference to a highly conserved sequence spanning bases 1017 to 1049 (5' CGTCGACTTC TTGTCCTCAATGACCACGC; HERVK-1017). For each enzyme analyzed, the estimated sizes for predicted HERVK-containing junction fragments were plotted on a log scale for comparison. Based on restriction fragment estimates with reference to genome coverage and the observed fragment distribution, $B s r \mathrm{I}$ was selected for unblot analysis and coordinate in silico comparison to the published sequence.

\section{Unblotting}

Unblotting, or hybridization in semi-dried agarose [14,58], was carried out to visualize polymorphic HERV-K proviruses within DNA samples. For each sample, $15 \mu \mathrm{g}$ of WGA DNA was digested with BsrI (New England Biolabs) in a $100 \mu \mathrm{L}$ volume and the digested products extracted and precipitated as described above. Products were resuspended in 20uL $0.25 \times \mathrm{TBE}+30 \%$ Ficol and electrophoresed through a $0.8 \%$ agarose gel in $0.25 \times \mathrm{TBE}$ at $70 \mathrm{~V}$ for $29 \mathrm{hr}$. at $4^{\circ} \mathrm{C}$. The gel was dehydrated in a vacuum dryer (BioRad) layered on filter papers for $60 \mathrm{~min}$. at RT and $60 \mathrm{~min}$. at $62^{\circ} \mathrm{C}$. The dried gel was stained with ethidium bromide in $0.25 \times \mathrm{TBE}$ and excess agarose removed with a clean scalpel. The gel was then incubated in denaturing buffer $(0.5 \mathrm{M} \mathrm{NaOH}+1.5 \mathrm{M} \mathrm{NaCl})$, and neutralizing buffer (1.0 M Tris- $\mathrm{HCl}+1.5 \mathrm{M} \mathrm{NaCl}, \mathrm{pH} 8.0) 30$ min. each at RT, and then hybridized with $7.5 \times 10^{6} \mathrm{cpm}$ of a ${ }^{32} \mathrm{P}$ labeled HERVK-1017 HML-2-specific oligonucleotide. Hybridization was in $5 \mathrm{~mL}$ of $5 \times \operatorname{SSPE}(3.0 \mathrm{M} \mathrm{NaCl}$, $0.2 \mathrm{M} \mathrm{NaH}_{2} \mathrm{PO}_{4}$, and $0.02 \mathrm{M}$ EDTA, pH 7.4) +0.1\% SDS, pH 7.4 at $53^{\circ} \mathrm{C}$ for $16 \mathrm{hr}$ with shaking at $50 \mathrm{rpm}$. Following hybridization, the gel was washed $(2 \times \mathrm{SSC}+$ $0.1 \%$ SDS) $4 \times$ for $15 \mathrm{~min}$. each at RT, and $2 \times$ for $30 \mathrm{~min}$. each at $53^{\circ} \mathrm{C}$ with shaking at $70 \mathrm{rpm}$. The gel was then exposed to BioMax MS film (Kodak) under an intensifying screen for $4-5$ days at $-70^{\circ} \mathrm{C}$.

\section{Statistical analyses}

Frequencies of individual sites were analyzed between case/control groups by $x^{2}$ analysis with one degree of 
freedom. For these analyses, comparisons were between cases and controls for individual polymorphic proviruses, calculated from 50 total samples ( 25 breast cancer per group). A $p$ value of less than 0.05 was taken to be significant. Total numbers of samples for scaled screening were determined by power analysis. For K115, to replicate a $20 \%$ difference between test groups with an $\alpha=0.05$, a statistical level of $80 \%$ power requires a sample size of $n=$ 94 (47 per cases and controls), and for $90 \%$ power a total sample size of $n=124$ (62 each group). All statistical analyses were performed by the Data Design and Resource Center at Tufts University.

\section{Abbreviations}

ACS: American Cancer Society; BLAT: BLAST-like alignment tool; CPSII: ACS Cancer Prevention II Nutritional Cohort; DNA: Deoxyribonucleic acid; ERV: Endogenous retrovirus; GWAS: Genome-wide association study; HERV: Human endogenous retrovirus; HML-2: Human MMTV-like; JSRV: Jaagsietke Sheep Retrovirus; LTR: Long terminal repeat; MMTV: Mouse Mammary Tumor Virus; ORF: Open reading frame; PCR: Polymerase chain reaction; RVLP: Retrovirus-like particle; SNP: Single nucleotide polymorphism; WGA: Whole genome amplification.

\section{Competing interests}

The authors declare no competing interests for this work.

\section{Authors' contributions}

$\mathrm{JHW}$ and JMC designed this study. JHW and JMC were responsible for data collection, analysis, and interpretation. JHW performed all necessary PCR, sequencing, and hybridization of genomic DNAs. JHW was also responsible for drafting and editing of this manuscript. DR was responsible for generation of 'in silico blotting' data based on the Hg19 build. JMC, VLS, and RS participated in the design and ongoing coordination of this work, and were involved in critical review and editing of the final manuscript. All authors have read and approved the final manuscript.

\section{Acknowledgements}

We are indebted to the American Cancer Society for providing the Cancer Prevention Cohort-II DNA samples utilized in this study. We thank the Tufts University Data Design and Resource Center for help with statistical analyses, and Jamie Henzy and Zachary Williams for meaningful discussion. This work was supported by Research Grant R37 CA 089441 from the National Cancer Institute to JMC. JHW was a recipient of a pre-doctoral NRSA Fellowship 5F31MH084487-02 from The National Institute for Mental Health. JMC was an American Cancer Society Research Professor with support from the FM Kirby Foundation.

\section{Author details}

'Department of Molecular Biology and Microbiology, Tufts University School of Medicine, 136 Harrison Avenue, Boston, MA 02111, USA. ${ }^{2}$ Department of Immunology, Tufts University School of Medicine, Boston, MA 02111, USA. ${ }^{3}$ Epidemiology Research Program, American Cancer Society, Atlanta, GA 30303, USA. ${ }^{4}$ Present address: Department of Human Genetics, The University of Michigan Medical School, 1241 E. Catherine St, Ann Arbor, MI 48109, USA.

Received: 30 May 2014 Accepted: 18 July 2014

Published: 12 August 2014

\section{References}

1. Howlader M, Noone AM, Krapcho M, Garsell J, Miler D, Altekruse SF, Kosary $\mathrm{CL}$, Yu M, Ruhl J, Tatlovich Z, Mariotto A, Lewis DR, Chen HS, Feuer EJ, Cronin KA: SEER Cancer Statistics Review (CSR) 1975-2011. Bethesda, MD: National Cancer Institute; 2014.

2. Siegel R, Naishadham D, Jemal A: Cancer statistics, 2013. CA Cancer J Clin 2013, 63:11-30.

3. HEK2 Breast Cancer Case-control Consortium: CHEK2*1100delC and susceptibility to breast cancer: a collaborative analysis involving 10,860 breast cancer cases and 9,065 controls from 10 studies. Am J Hum Genet 2004, 74:1175-1182.

4. Easton DF, Pooley KA, Dunning AM, Pharoah PD, Thompson D, Ballinger DG, Struewing JP, Morrison J, Field H, Luben R, Wareham N, Ahmed S, Healey CS, Bowman R, Meyer KB, Haiman CA, Kolonel LK, Henderson BE, Le Marchand L, Brennan P, Sangrajrang S, Gaborieau V, Odefrey F, Shen CY, Wu PE, Wang HC, Eccles D, Evans DG, Peto J, Fletcher O, et al: Genome-wide association study identifies novel breast cancer susceptibility loci. Nature 2007, 447:1087-1093.

5. Renwick A, Thompson D, Seal S, Kelly P, Chagtai T, Ahmed M, North B, Jayatilake H, Barfoot R, Spanova K, McGuffog L, Evans DG, Eccles D, Easton DF, Stratton MR, Rahman N: ATM mutations that cause ataxia-telangiectasia are breast cancer susceptibility alleles. Nat Genet 2006, 38:873-875.

6. Wooster R, Weber BL: Breast and ovarian cancer. N Engl J Med 2003, 348:2339-2347.

7. Meijers-Heijboer H, van den Ouweland A, Klijn J, Wasielewski M, de Snoo A, Oldenburg R, Hollestelle A, Houben M, Crepin E, van Veghel-Plandsoen M, Elstrodt F, van Duijn C, Bartels C, Meijers C, Schutte M, McGuffog L, Thompson D, Easton D, Sodha N, Seal S, Barfoot R, Mangion J, Chang-Claude J, Eccles D, Eeles R, Evans DG, Houlston R, Murday V, Narod S, Peretz T, et al: Low-penetrance susceptibility to breast cancer due to CHEK2 $(*) 1100 \mathrm{del} C$ in noncarriers of BRCA1 or BRCA2 mutations. Nat Genet 2002, 31:55-59.

8. Rahman N, Seal S, Thompson D, Kelly P, Renwick A, Elliott A, Reid S, Spanova K, Barfoot R, Chagtai T, Jayatilake H, McGuffog L, Hanks S, Evans DG, Eccles D, Easton DF, Stratton MR: PALB2, which encodes a BRCA2interacting protein, is a breast cancer susceptibility gene. Nat Genet 2007, 39:165-167.

9. Seal $S$, Thompson D, Renwick A, Elliott A, Kelly P, Barfoot R, Chagtai T, Jayatilake H, Ahmed M, Spanova K, North B, McGuffog L, Evans DG, Eccles D, Easton DF, Stratton MR, Rahman N: Truncating mutations in the Fanconi anemia J gene BRIP1 are low-penetrance breast cancer susceptibility alleles. Nat Genet 2006, 38:1239-1241.

10. Ripperger T, Gadzicki D, Meindl A, Schlegelberger B: Breast cancer susceptibility: current knowledge and implications for genetic counselling. Eur J Hum Genet 2009, 17:722-731.

11. IHGSC: A physical map of the human genome. Nature 2001, 409:934-941.

12. Boeke JD, Stoye JP: Retrotransposons, endogenous retroviruses, and the evolution of retroelements. In Retroviruses. Edited by Hughes S, Varmus H. Cold Spring Harbor, NY: Cold Spring Harbor Laboratory Press; 1997:343-435.

13. Jern P, Coffin JM: Effects of retroviruses on host genome function. Annu Rev Genet 2008, 42:709-732.

14. Stoye J, Frankel W, Coffin J: DNA hybridization in dried gels with fragmented probes:an improvement over blotting techniques. Technique 1991, 3:123-128.

15. Barbulescu M, Turner G, Seaman MI, Deinard AS, Kidd KK, Lenz J: Many human endogenous retrovirus $\mathrm{K}$ (HERV-K) proviruses are unique to humans. Curr Biol 1999, 9:861-868.

16. Belshaw R, Dawson AL, Woolven-Allen J, Redding J, Burt A, Tristem M: Genomewide screening reveals high levels of insertional polymorphism in the human endogenous retrovirus family HERV-K(HML2): implications for present-day activity. J Virol 2005, 79:12507-12514.

17. Hughes JF, Coffin JM: Human endogenous retrovirus K solo-LTR formation and insertional polymorphisms: implications for human and viral evolution. Proc Natl Acad Sci U S A 2004, 101:1668-1672.

18. Medstrand P, Mager DL: Human-specific integrations of the HERV-K endogenous retrovirus family. J Virol 1998, 72:9782-9787.

19. Subramanian RP, Wildschutte JH, Russo C, Coffin JM: Identification, characterization, and comparative genomic distribution of the HERV-K (HML-2) group of human endogenous retroviruses. Retrovirology 2011, 8:90.

20. Turner G, Barbulescu M, Su M, Jensen-Seaman MI, Kidd KK, Lenz J: Insertional polymorphisms of full-length endogenous retroviruses in humans. Curr Biol 2001, 11:1531-1535.

21. Belshaw R, Pereira V, Katzourakis A, Talbot G, Paces J, Burt A, Tristem M: Long-term reinfection of the human genome by endogenous retroviruses. Proc Natl Acad Sci U S A 2004, 101:4894-4899.

22. Beimforde N, Hanke K, Ammar I, Kurth R, Bannert N: Molecular cloning and functional characterization of the human endogenous retrovirus $\mathrm{K} 113$. Virology 2008, 371:216-225.

23. Boller K, Schonfeld K, Lischer S, Fischer N, Hoffmann A, Kurth R, Tonjes RR: Human endogenous retrovirus HERV-K113 is capable of producing intact viral particles. J Gen Virol 2008, 89:567-572. 
24. Flockerzi A, Ruggieri A, Frank O, Sauter M, Maldener E, Kopper B, Wullich B, Seifarth W, Muller-Lantzsch N, Leib-Mosch C, Meese E, Mayer J: Expression patterns of transcribed human endogenous retrovirus HERV-K(HML-2) loci in human tissues and the need for a HERV Transcriptome Project. BMC Genomics 2008, 9:354.

25. Seifarth W, Frank O, Zeilfelder U, Spiess B, Greenwood AD, Hehlmann R, Leib-Mosch C: Comprehensive analysis of human endogenous retrovirus transcriptional activity in human tissues with a retrovirus-specific microarray. J Virol 2005, 79:341-352.

26. Costas J: Evolutionary dynamics of the human endogenous retrovirus family HERV-K inferred from full-length proviral genomes. $J \mathrm{Mol}$ Evol 2001, 53:237-243.

27. Reus K, Mayer J, Sauter M, Scherer D, Muller-Lantzsch N, Meese E: Genomic organization of the human endogenous retrovirus HERV-K(HML-2.HOM) (ERVK6) on chromosome 7. Genomics 2001, 72:314-320.

28. Dewannieux M, Harper F, Richaud A, Letzelter C, Ribet D, Pierron G, Heidmann $\mathrm{T}$ : Identification of an infectious progenitor for the multiple-copy HERV-K human endogenous retroelements. Genome Res 2006, 16:1548-1556.

29. Lee YN, Bieniasz PD: Reconstitution of an infectious human endogenous retrovirus. PLoS Pathog 2007, 3:e10.

30. Wang-Johanning F, Liu J, Rycaj K, Huang M, Tsai K, Rosen DG, Chen DT, Lu DW, Barnhart KF, Johanning GL: Expression of multiple human endogenous retrovirus surface envelope proteins in ovarian cancer. Int J Cancer 2007, 120:81-90.

31. Boller K, Konig H, Sauter M, Mueller-Lantzsch N, Lower R, Lower J, Kurth R: Evidence that HERV-K is the endogenous retrovirus sequence that codes for the human teratocarcinoma-derived retrovirus HTDV. Virology 1993, 196:349-353.

32. Herbst H, Sauter M, Kuhler-Obbarius C, Loning T, Mueller-Lantzsch N: Human endogenous retrovirus (HERV)-K transcripts in germ cell and trophoblastic tumours. APMIS 1998, 106:216-220.

33. Lower R, Lower J, Frank H, Harzmann R, Kurth R: Human teratocarcinomas cultured in vitro produce unique retrovirus-like viruses. J Gen Virol 1984, 65(Pt 5):887-898.

34. Ruprecht K, Ferreira H, Flockerzi A, Wahl S, Sauter M, Mayer J, MuellerLantzsch N: Human endogenous retrovirus family HERV-K(HML-2) RNA transcripts are selectively packaged into retroviral particles produced by the human germ cell tumor line Tera- 1 and originate mainly from a provirus on chromosome 22q11.21. J Virol 2008, 82:10008-10016.

35. Buscher K, Hahn S, Hofmann M, Trefzer U, Ozel M, Sterry W, Lower J, Lower $\mathrm{R}$, Kurth R, Denner J: Expression of the human endogenous retrovirus-K transmembrane envelope, Rec and Np9 proteins in melanomas and melanoma cell lines. Melanoma Res 2006, 16:223-234.

36. Buscher K, Trefzer U, Hofmann M, Sterry W, Kurth R, Denner J: Expression of human endogenous retrovirus $\mathrm{K}$ in melanomas and melanoma cell lines. Cancer Res 2005, 65:4172-4180.

37. Hahn S, Ugurel S, Hanschmann KM, Strobel H, Tondera C, Schadendorf D, Lower J, Lower R: Serological response to human endogenous retrovirus $\mathrm{K}$ in melanoma patients correlates with survival probability. AIDS Res Hum Retroviruses 2008, 24:717-723.

38. Mangeney M, Pothlichet J, Renard M, Ducos B, Heidmann T: Endogenous retrovirus expression is required for murine melanoma tumor growth in vivo. Cancer Res 2005, 65:2588-2591.

39. Muster T, Waltenberger A, Grassauer A, Hirschl S, Caucig P, Romirer I, Fodinger D, Seppele H, Schanab O, Magin-Lachmann C, Lower R, Jansen B, Pehamberger $\mathrm{H}$, Wolff $\mathrm{K}$ : An endogenous retrovirus derived from human melanoma cells. Cancer Res 2003, 63:8735-8741.

40. Singh S, Kaye S, Francis N, Peston D, Gore M, McClure M, Bunker C: Human endogenous retrovirus $K$ (HERV-K) rec mRNA is expressed in primary melanoma but not in benign naevi or normal skin. Pigment Cell Melanoma Res 2013, 26:426-428.

41. Contreras-Galindo R, Kaplan MH, Leissner P, Verjat T, Ferlenghi I, Bagnoli F, Giusti F, Dosik MH, Hayes DF, Gitlin SD, Markovitz DM: Human endogenous retrovirus $\mathrm{K}(\mathrm{HML}-2)$ elements in the plasma of people with lymphoma and breast cancer. J Virol 2008, 82:9329-9336.

42. Iwabuchi $H$, Kakihara $T$, Kobayashi $T$, Imai C, Tanaka A, Uchiyama M, Fukuda $\mathrm{T}$ : A gene homologous to human endogenous retrovirus overexpressed in childhood acute lymphoblastic leukemia. Leuk Lymphoma 2004 45:2303-2306.

43. Armbruester V, Sauter M, Krautkraemer E, Meese E, Kleiman A, Best B, Roemer $\mathrm{K}$, Mueller-Lantzsch N: A novel gene from the human endogenous retrovirus $\mathrm{K}$ expressed in transformed cells. Clin Cancer Res 2002, 8:1800-1807.

44. Frank O, Verbeke C, Schwarz N, Mayer J, Fabarius A, Hehlmann R, Leib-Mosch C, Seifarth W: Variable transcriptional activity of endogenous retroviruses in human breast cancer. J Virol 2008, 82:1808-1818.

45. Ono M, Kawakami M, Ushikubo H: Stimulation of expression of the human endogenous retrovirus genome by female steroid hormones in human breast cancer cell line T47D. J Virol 1987, 61:2059-2062.

46. Wang-Johanning F, Frost AR, Jian B, Epp L, Lu DW, Johanning GL: Quantitation of HERV-K env gene expression and splicing in human breast cancer. Oncogene 2003, 22:1528-1535.

47. Wang-Johanning F, Frost AR, Johanning GL, Khazaeli MB, LoBuglio AF, Shaw $D R$, Strong TV: Expression of human endogenous retrovirus $k$ envelope transcripts in human breast cancer. Clin Cancer Res 2001, 7:1553-1560.

48. Willer A, Saussele S, Gimbel W, Seifarth W, Kister P, Leib-Mosch C, Hehlmann $R$ : Two groups of endogenous MMTV related retroviral env transcripts expressed in human tissues. Virus Genes 1997, 15:123-133.

49. Faff $O$, Murray AB, Schmidt J, Leib-Mosch C, Erfle V, Hehlmann R: Retroviruslike particles from the human T47D cell line are related to mouse mammary tumour virus and are of human endogenous origin. $J$ Gen Virol 1992, 73(Pt 5):1087-1097.

50. Seifarth W, Baust C, Murr A, Skladny H, Krieg-Schneider F, Blusch J, Werner T, Hehlmann R, Leib-Mosch C: Proviral structure, chromosomal location, and expression of HERV-K-T47D, a novel human endogenous retrovirus derived from T47D particles. J Virol 1998, 72:8384-8391.

51. Seifarth W, Skladny H, Krieg-Schneider F, Reichert A, Hehlmann R, Leib-Mosch $C$ : Retrovirus-like particles released from the human breast cancer cell line T47-D display type B- and C-related endogenous retroviral sequences. J Virol 1995, 69:6408-6416.

52. Hook LM, Agafonova Y, Ross SR, Turner SJ, Golovkina TV: Genetics of mouse mammary tumor virus-induced mammary tumors: linkage of tumor induction to the gag gene. J Virol 2000, 74:8876-8883.

53. Salmons B, Gunzburg WH: Current perspectives in the biology of mouse mammary tumour virus. Virus Res 1987, 8:81-102.

54. Burmeister T, Ebert AD, Pritze W, Loddenkemper C, Schwartz S, Thiel E: Insertional polymorphisms of endogenous HERV-K113 and HERV-K115 retroviruses in breast cancer patients and age-matched controls. AIDS Res Hum Retroviruses 2004, 20:1223-1229.

55. Krzysztalowska-Wawrzyniak M, Ostanek M, Clark J, Binczak-Kuleta A, Ostanek L, Kaczmarczyk M, Loniewska B, Wyrwicz LS, Brzosko M, Ciechanowicz A: The distribution of human endogenous retrovirus K-113 in health and autoimmune diseases in Poland. Rheumatology (Oxford) 2010, 7:13-21.

56. Moyes DL, Goris A, Ban M, Compston A, Griffiths DJ, Sawcer S, Venables PJ: HERV-K113 is not associated with multiple sclerosis in a large family-based study. AlDS Res Hum Retroviruses 2008, 24:363-365.

57. Moyes DL, Martin A, Sawcer S, Temperton N, Worthington J, Griffiths DJ, Venables PJ: The distribution of the endogenous retroviruses HERV-K113 and HERV-K115 in health and disease. Genomics 2005, 86:337-341.

58. Frankel WN, Stoye JP, Taylor BA, Coffin JM: A linkage map of endogenous murine leukemia proviruses. Genetics 1990, 124:221-236

59. Calle EE, Rodriguez C, Jacobs EJ, Almon ML, Chao A, McCullough ML, Feigelson HS, Thun MJ: The American Cancer Society Cancer Prevention Study II Nutrition Cohort: rationale, study design, and baseline characteristics. Cancer 2002, 94:500-511.

60. Otowa T, Tochigi M, Rogers M, Umekage T, Kato N, Sasaki T: Insertional polymorphism of endogenous retrovirus HERV-K115 in schizophrenia. Neurosci Lett 2006, 408:226-229.

doi:10.1186/s12977-014-0062-3

Cite this article as: Wildschutte et al:: The distribution of insertionally polymorphic endogenous retroviruses in breast cancer patients and cancer-free controls. Retrovirology 2014 11:62. 